\title{
Collaborative control of common-mode voltage suppression and neutral-point potential balance based on carrier modulation for non-isolation V2G system*
}

\author{
Xiang-Wu Yan ${ }^{1}$, Zhi-Heng Xiao ${ }^{1,2, \dagger}$ and Jia-Le Zhao ${ }^{1}$ \\ ${ }^{I}$ State Key Laboratory of Alternate Electrical Power System \\ with Renewable Energy Sources, North China Electric Power University \\ Baoding, 071003, China \\ ${ }^{2}$ North China Electric Power University \\ Baoding, 071003, China \\ †E-mail:youzhichiheng@163.com
}

\begin{abstract}
For problems that there is common-mode voltage in non-isolation three-phase three-level V2G integration system, the common-mode equivalent model was established. Meanwhile, it revealed that DC link neutral-point potential imbalance would limit the effect of common-mode voltage suppression. The analysis revealed that in-phase disposition modulation would lead to larger fluctuations in the common-mode voltage, although the neutral-point potential is balanced by injecting zero-sequence voltage, it has no effect on the suppression of common-mode voltage; The opposite phase disposition modulation could reduce common-mode voltage peak value, but zero-sequence voltage injection is not suitable for which to balance neutral-point potential. Furthermore, we propose a new modulation strategy of bipolar carrier modulation combined with the virtual space vector modulation $\left(\mathrm{NTV}^{2}\right)$, keeping the output of common-mode voltage be constant; meanwhile, we imported $\mathrm{NTV}^{2}$ to redefine the virtual medium vector to keep neutral-point potential balance in full range. Thereby the new modulation strategy achieved the collaborative control of the elimination of common-mode current and neutral-point potential balance. Finally, we took the respective simulation research to the new modulation strategy. The simulation results demonstrated the effectiveness of collaborative control strategy.
\end{abstract}

Keywords: Non-isolation V2G, Carrier modulation, Common-mode voltage suppression, Neutral-point potential balance, Collaborative control.

\section{Introduction}

Non-isolation three-phase three-level topology is now the mainly international trend for $\mathrm{V} 2 \mathrm{G}$ integration system, which has the advantages of simple structure, small volume, low cost and high efficiency [1]. Integrated V2G system schematic

\footnotetext{
* This research was financially supported by National High-tech R\&D Program (863 Program) of China(2015AA050603) and Science and Technology Program of State Grid (SGTYHT/14-JS-188)
} 
diagram is shown in figure 1 . Where $U_{p v}$ is the output voltage of the electric vehicle battery? The parasitic capacitance between the switch tube of the upper and lower bridge arm and the heat sink is $C_{h}$ and $C_{s m p}, C_{p v}$ represents the parasitic capacitance between the batteries and car body(ground).

However, saving the transformer takes the direct impact that there are electrical connections between integrated V2G system and power grid. The formation of the common-mode current will bring security risks, but also affect the normal use of equipment, accelerate the equipment aging[1, 2]. Therefore, the suppression of common-mode voltage is the prime problem [3].

Compared with the space vector modulation, the carrier modulation has the characteristics of simple principle and easy to implement. Literature [4, 5] analyzed how different modulation strategies influenced the leakage current of the non-isolation three-level photovoltaic inverter, but it did not take account of three-level system inherent neutral-point potential balancing problems. Literature $[6,7]$ adopted the common neutral-point potential balance control methodinjecting zero sequence voltage. However, it can't take into account the common-mode voltage suppression problem, making it too simplified.

In this paper, the influence of the traditional in-phase disposition and opposite phase disposition modulation on the system common-mode voltage is discussed. And the corresponding neutral point potential balancing method is presented respectively. Then we put forward a new carried modulation strategy.

\section{The Common-Mode Equivalent Model of Non-Isolation V2G System}

The equivalent model of common-mode circuit of Figure 1(b) is established. [6]. $U_{c m}=U_{c m 1}+U_{c m 2}$, For AC side, according to Kirchhoff's law:

$$
U_{c m 1}=\frac{U_{A Q}+U_{B Q}+U_{C Q}}{3}
$$

For DC side:

$$
U_{c m 2}=\frac{C_{s m p}}{C_{s m p}+C_{p v}} U_{N Q}
$$



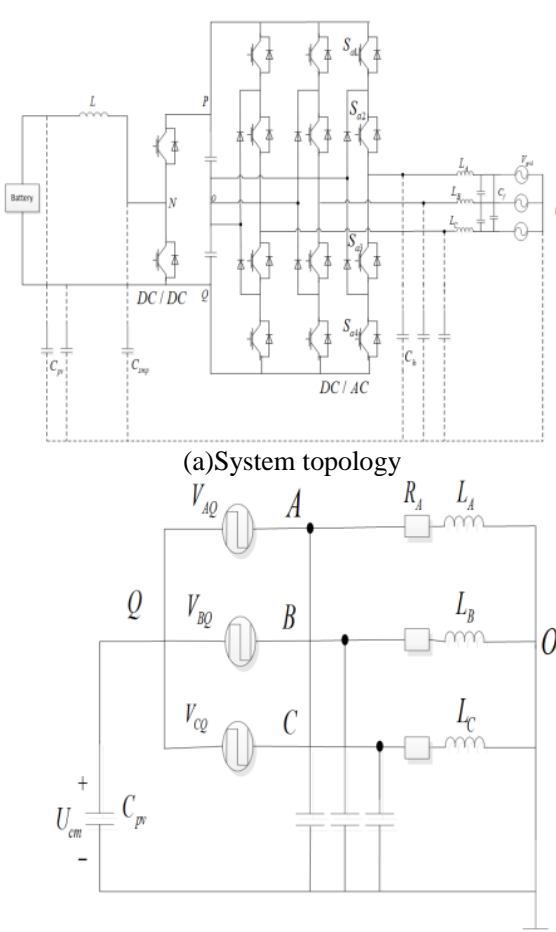

(c) AC side equivalent circuit

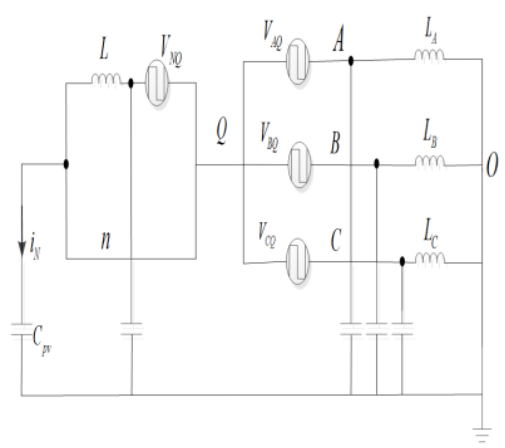

(b) CM equivalent model

Fig.1. Common-mode equivalent model of non-isolation V2G system

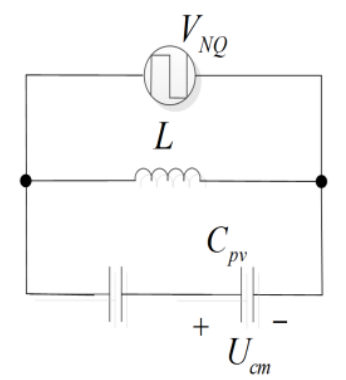

(d) DC side

$\mathrm{U}_{\mathrm{cm} 2}$ is no more than $\pm 1 V$, that is to say, the contribution of the front-end DC/DC converter can be ignored. Then the effects of different carrier modulation

Strategies on the common mode voltage are analyzed.

\section{In-phase Disposition Modulation}

Three-phase positive reference voltage (normalized) steady-state expression is as follows, Eq. (4) is also three-phase modulation wave expression [7].

$$
\left\{\begin{array}{l}
v_{r a}(t)=M \cos w t \\
v_{r b}(t)=M \cos (w t-2 \pi / 3) \\
v_{r c}(t)=M \cos (w t+2 \pi / 3)
\end{array}\right.
$$




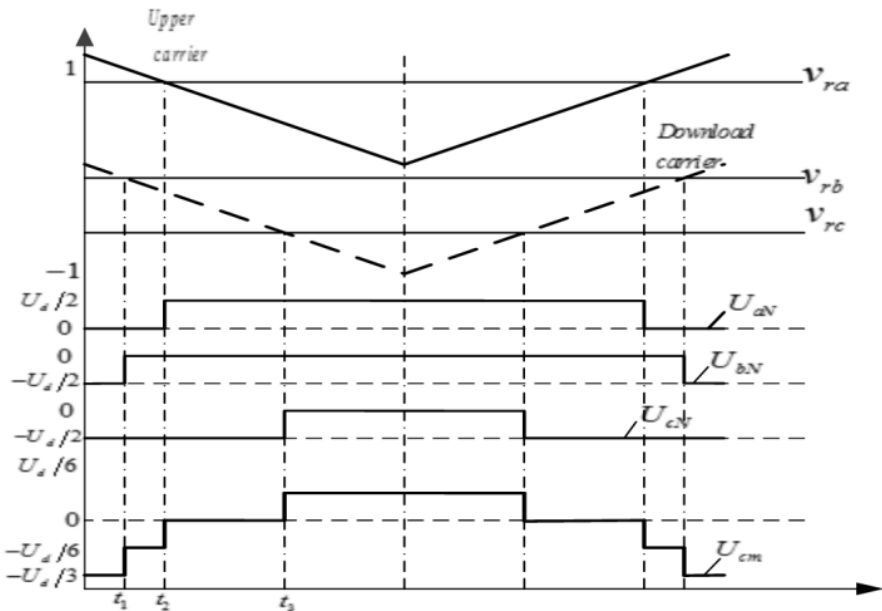

(a) Schematic

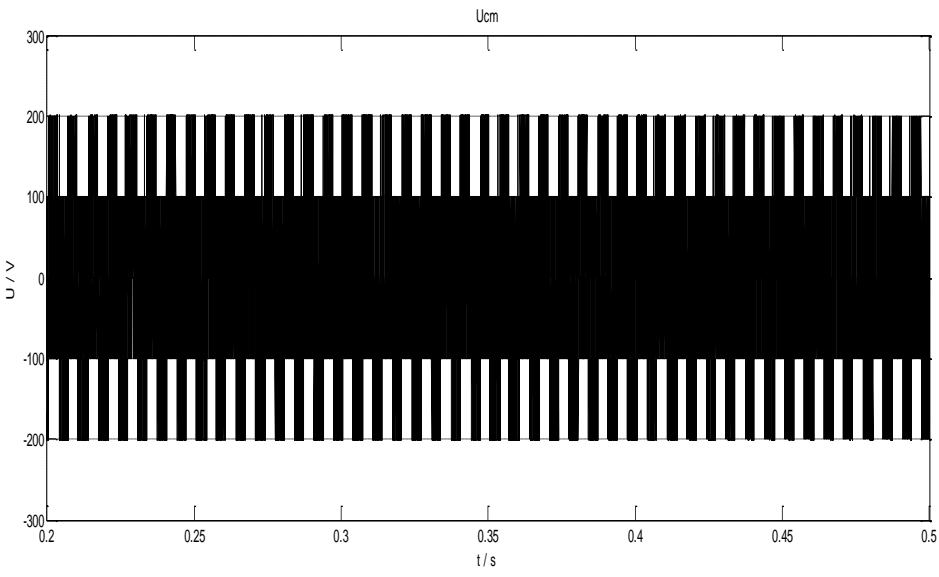

(b) CM (common-mode) voltage

Fig. 2. Schematic of in-phase disposition modulation

Figure 2 shows the schematic of in-phase disposition modulation. The common-mode voltage peak value of the system is $U_{d c} / 3$.

In-phase disposition modulation can balance the neutral-point potential by injecting zero sequence voltage [8], as the graphic condition shown in figure 4, the method is essentially by judging the migration direction of the neutral-point potential, then adjust the action time of the positive and negative redundant small vector, which has no effect on the suppression of the common-mode voltage. 


\section{Opposite Phase Disposition Modulation}

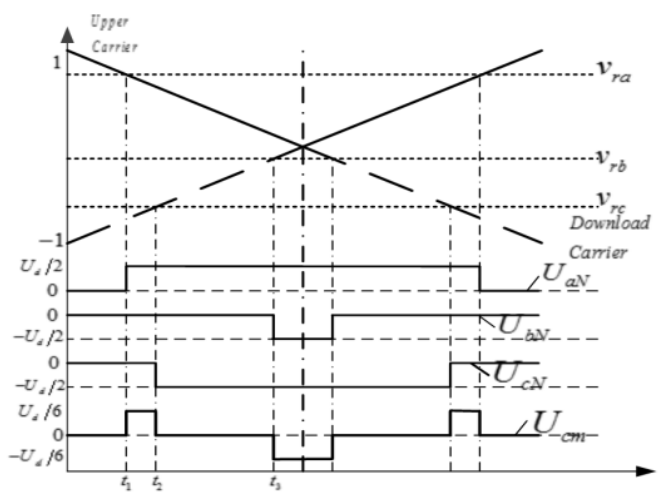

(a) Schemati

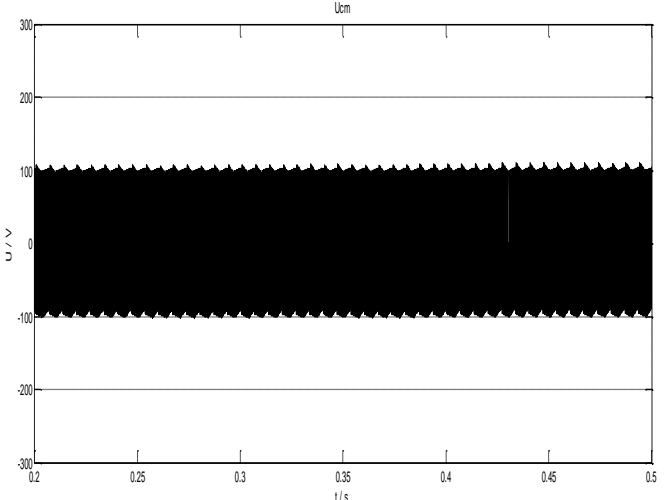

(b) CM voltage

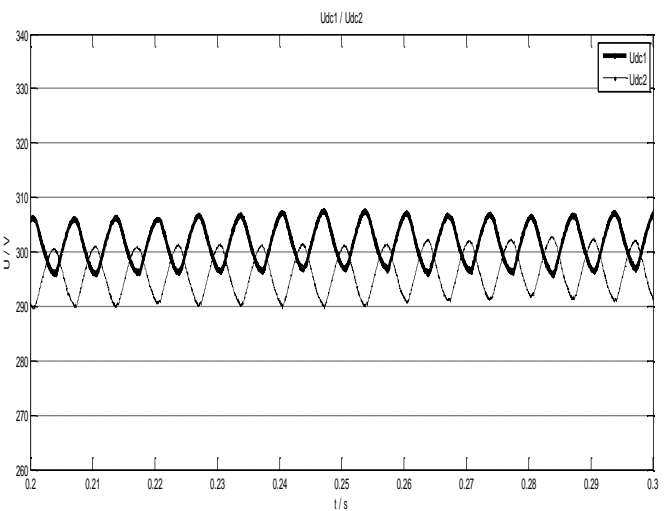

(c) DC capacitor voltage $u_{d c 1} / u_{d c 2}$

Fig. 3. Schematic of opposite phase disposition modulation.

The schematic is shown in Figure 3. As shown in Figure 3 (c), it can't control 
the balance of neutral-point potential well. Through analysis, the redundant small vector pairs will not occur at the same time when opposite phase disposition modulation is taken [9]. Therefore, the neutral-point potential balance can't be achieved by injecting zero sequence voltage.

\section{Novel Carried Modulation Strategy for Collaborative Control}

A new carried modulation scheme based on Boolean logic operation is proposed in this paper, on the basis of Boolean logic operation, the mapping from the switching state of the carrier modulation to the specific space vector is established, realizing the corresponding relation in Table 1 [8]

Table 1. Switching logic states

\begin{tabular}{cccc}
\hline$A B C$ & Corresponding vector & $A B C$ & Corresponding vector \\
010 & NPO & 101 & PNO \\
110 & OPN & 100 & PON \\
011 & NOP & 000 & OOO \\
001 & ONP & 111 & OOO \\
\hline
\end{tabular}

$$
S_{a}=\mathrm{A}-\mathrm{B}, S_{b}=\mathrm{B}-\mathrm{C}, S_{c}=\mathrm{C}-\mathrm{A}
$$

Boolean logic operation is shown in Eq. (4). The proposed carried modulation scheme only needs to add digital logic in the bipolar modulation, and can realize the switch status in Table 2. On the other hand, we introduce the virtual vector method and optimize the define of virtual medium vector $V_{m}^{\prime}$, which using three adjacent basic medium vector to synthesis as Eq. (5)
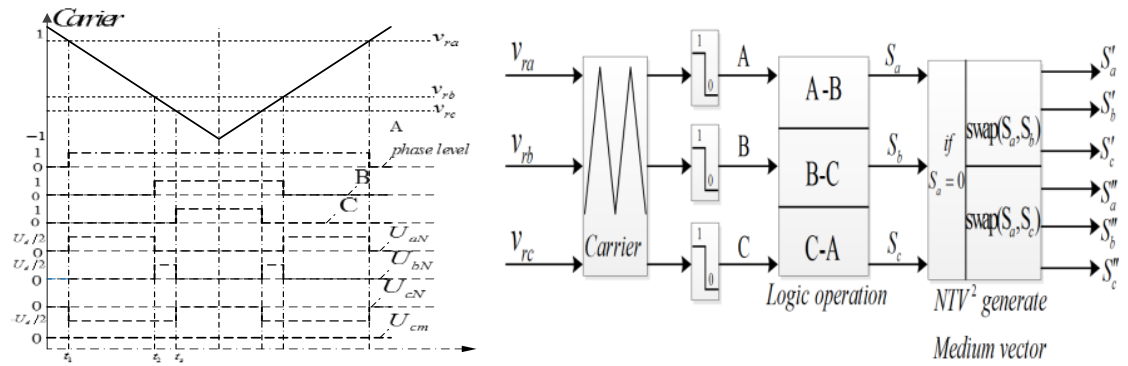

Fig. 5. Schematic of Bipolar modulation. Fig. 6. Proposed new carrier-based modulation solution.

$$
V_{m}^{\prime}=\frac{1}{3} V_{m(\mathrm{PNO})}+\frac{1}{3} V_{m(\mathrm{PON})}+\frac{1}{3} V_{m(\mathrm{OPN})}
$$

The virtual medium vector re-definition and zero vector can ensure the production of neutral current is zero, Therefore, SPWM combined with $\mathrm{NTV}^{2}$ method can control the neutral-point potential balance in the whole range [9].On 
the basis of the Eq. (4), we can continue to determine the adjacent two basic medium vectors by Boolean operation: firstly, choose one from $S_{a}, S_{b}, S_{c}$ which corresponding switching state is zero, after just exchanging the numerical value of the other two phases with zero phase respectively.

\section{Simulation Results}

Based on MATLAB/SIMULINK platform, we established a non-isolated Three-phase three-level V2G system model, the parameters are as follows: $L_{A}=L_{B}=L_{C}=3.5 \mathrm{mH}, C_{1}=C_{2}=3 \mathrm{mH}, C_{p v}=2 \mathrm{nF} ; \mathrm{DC} / \mathrm{DC}$ and DC/AC parts both adopted double closed-loop control. Unified switching frequency into $f_{S}=10 \mathrm{kHz}$, Request the DC bus voltage $U_{d c}$ is required stabilized at $600 \mathrm{~V}$.

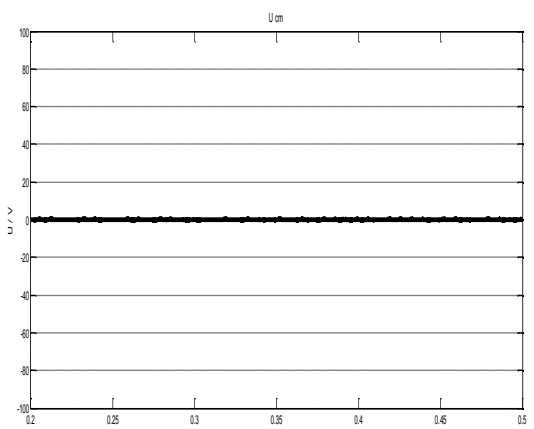

(a)CM voltage

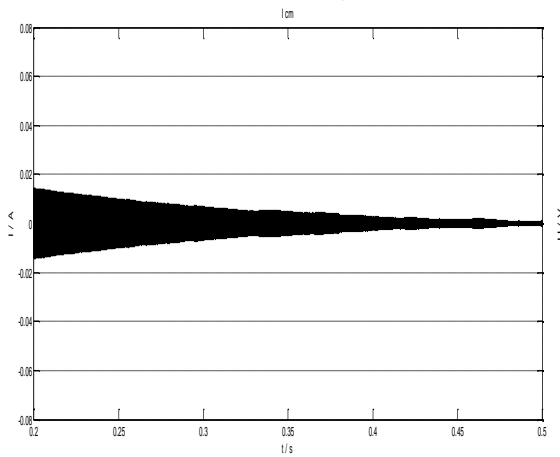

(c) Amplification of CM current

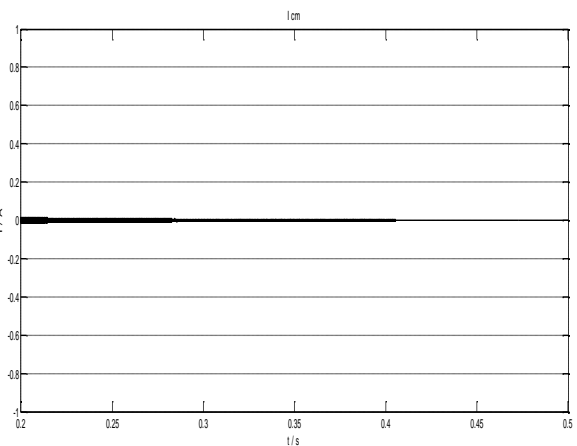

(b) CM current

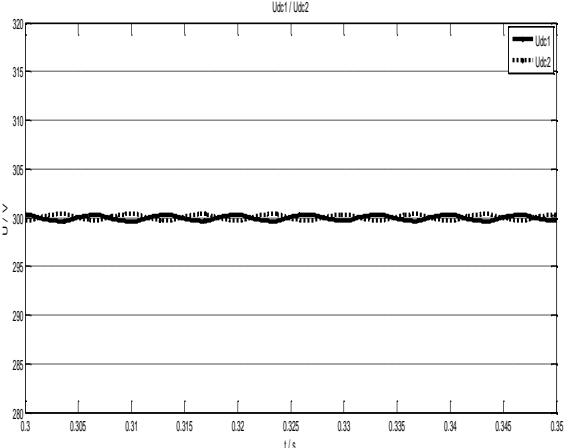

(d) DC capacitor $u_{d c 1} / u_{d c 2}$

Fig.7. Simulation waveform of the system with new carrier-based modulation strategy

Figure 7 shows the simulation waveform of the system with new carrier-based modulation strategy. Combining with the above figure, we can find that the common-mode voltage keep mainly constant when using the new carrier modulation strategy, so the common-mode current is very small, its value is less 
than $20 \mathrm{~mA}$. The neutral voltage fluctuation range is small, which can prove that the strategy can control neutral-point potential balance in full range.

\section{Conclusion}

This paper deeply discusses the common-mode voltage suppression of the non isolated three-phase three-level V2G system,

(1)The common mode equivalent model is established, the excitation source of common mode voltage is determined;

(2)Although in-phase disposition modulation method leads to larger common-mode voltage fluctuation, it can balance neutral-point potential by injecting zero sequence voltage;

(3)The opposite phase disposition modulation can reduce the amplitude of the common-mode voltage by half, from $U_{d c} / 3$ to $U_{d c} / 6$. Meanwhile; it can't balance the neutral-point potential by injecting zero sequence voltage.

(4)We propose a new modulation scheme combined bipolar modulation, which can eliminate common-mode voltage and balance neutral voltage at the same time.

Based on MATLAB/SIMULINK platform, the results confirmed that the method proposed in this paper have obvious advantages on the common-mode voltage suppression and neutral-point potential balance in DC side. According to different application occasions, the staff can flexibly choose the appropriate collaborative modulation strategy.

\section{References}

1. O. Hegazy, J. Van Mierlo, and P. Lataire(2011) Design and control of bidirectional DC/AC and DC/DC converters for plug-in hybrid electric vehicles. Paper presented at the Power Engineering, Energy and Electrical Drives, International Conference on, May 2011, pp. 1-7.

2. Wu Weiyang, Guo Xiao-qiang(2012). A review of novel leakage current suppression techniques for transformer less photovoltaic inverters. Proceedings of the CSEE.32 (18):1-8(in Chinese).

3. Guo Xiao-qiang, He ran, Zhang Chun-jiang, et al (2016).Common-mode Voltage Proceedings of the CSEE. v.3103:1-7.

4. Su Na (2012) Analysis and suppression of leakage current in PV Inverter. Zhejiang University (in Chinese).

5. ZHANG Xing ,SHAO Zhangping, WANG Fu-sheng, LIU Ping, et al(2013)Leakage Current Reduction for Transformer less Three-phase Three-level Photovoltaic Inverters. Proceedings of the CSEE.03:29-36+18. 
6. Xiao Huafeng, Xie Shaojun, Chen Wenming, et al (2010) Study on leakage current model for tansformerless photovoltaic grid-connected inverter. Proceedings of the CSEE.30 (18):9-14(in Chinese).

7. GUO Xiaoping, LI Jian, WEI Baoze, et al (2015) Analysis of Carrier-based Modulation for Leakage Currents in Transformer less Three-level Photovoltaic Inverters. Proceedings of the CSEE.v.35; No. 53116:4167-4174.

8. Wu Keli, Xia Changliang, Zhang Yun, et al (2015) Common-Mode Voltage Suppression for Neutral-Point Clamped Three-Level Inverter. Transactions of China Electro technical Society.24: 110-117+170.

9. GUI Shi-weng, WU Fang, WAN Shan-ming, et al (2015) A Strategy for Considering Neutral-point Potential Balance for Three-level NPC Inverters with the Varied Virtual Space Vector. Transactions of China Electro technical Society.v.35; No.53419:5013-5021(in Chinese). 